\title{
Freegaming: Mobile, collaborative, adaptive and augmented exergaming
}

\author{
Levent Görgü̈а, ${ }^{\mathrm{a}, *}$ Abraham G. Campbell ${ }^{\mathrm{a}}$, Kealan McCusker ${ }^{\mathrm{b}}$, Mauro Dragone ${ }^{\mathrm{a}}$, \\ Michael J. O'Grady ${ }^{\mathrm{a}}$, Noel E. O'Connor ${ }^{\mathrm{b}}$ and Gregory M.P. O'Hare ${ }^{\mathrm{a}}$ \\ ${ }^{a}$ CLARITY: Centre for Sensor Web Technologies, University College Dublin, Dublin, Ireland \\ ${ }^{\mathrm{b}}$ CLARITY: Centre for Sensor Web Technologies, Dublin City University, Dublin, Ireland
}

\begin{abstract}
Addressing the obesity epidemic that plagues many societies remains an outstanding public health issue. One innovative approach to addressing this problem is Exergaming. A combination of Exercise and Gaming, the objective is to motivate people participate in exercise regimes, usually in their home environment. In this article a more holistic interpretation of this exercise paradigm is proposed. Freegaming augments Exergaming in a number of key dimensions but especially through the promotion of games in outdoor mobile contexts and within a social environment. The design and implementation of a platform for Freegaming is described and illustrated through the description of a sample game.
\end{abstract}

Keywords: Mobile multimedia, augmented reality, exergaming, pervasive health, multiagent systems

\section{Introduction}

In recent years, childhood obesity has become a major problem in many countries. According to several studies $[28,48]$, obese people have an increased risk for many health conditions, for example, coronary heart disease, type 2 diabetes, different types of cancer, certain musculoskeletal disorders, such as knee osteoarthritis, and also for many chronic conditions such as hypertension and elevated cholesterol levels. At the same time, overweight children can more easily become overweight adults [1, 28]. There are approximately 17.6 million children worldwide aged less than five which are estimated to be overweight [41]. Most of the research about the treatment of obesity recommends an increase in physical activity combined with a controlled low calorie diet $[28,42,48,49]$. Nutrition researchers at UNC-Chapel Hill surprisingly demonstrated that the lack of physical activity, but not increased energy intake, is also responsible for obesity among youths aged 12-19 [40]. Video games are considered the main reason for physical inactivity [44]. Such games do not encourage any serious physical effort, and are generally blamed for consuming time from the younger population which could be spent in physical activities like playing out-doors. Also, many of these games do not need any collaboration, and the players generally prefer to play them alone, which contributes to antisocial children [36].

The exergaming concept [7] searches for ways to combine exercising and gaming by disguising the tiresome side of working out with the uplifting side of playing to make the exercise process more attractive, not only for the younger population but also for anybody suffering from physical exercise

\footnotetext{
${ }^{*}$ Corresponding author: Levent Görgü, Centre for Sensor Web Technologies, University College Dublin, Belfield, Dublin 4, Ireland. Tel.: +353 1716 2818; E-mail: levent.gorgu@ucd.ie.
} 

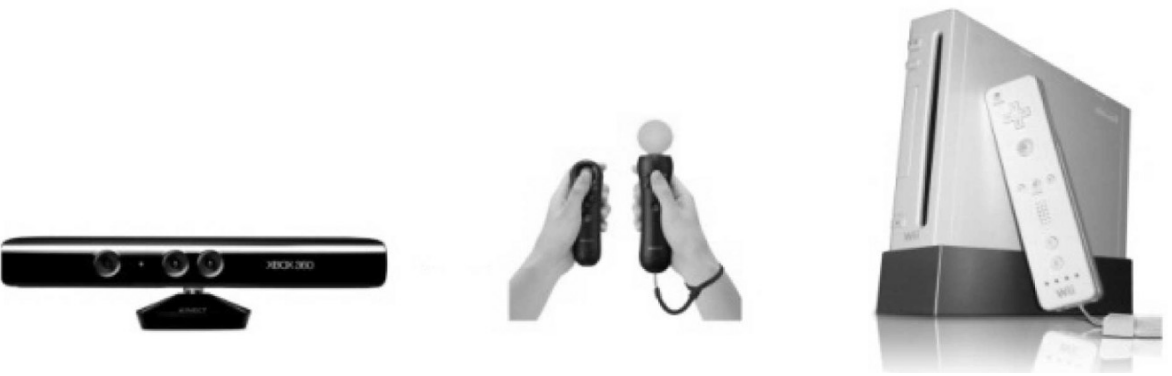

Fig. 1. Microsoft Kinect, Sony PlayStation Move and Nintendo Wii - Indoor exergaming systems for the most popular three game consoles on the market.

deprivation [26]. Thus, mobile collaborative exergaming forces players to collaborate and communicate with each other, while implicitly encouraging them to increase their physical activity levels while playing but without inducing boredom. Optimistic results about increased physical activity levels have been demonstrated from playing games [16], but all these systems are console dependent. On the other hand, studies [45] show that the mobile game market is estimated to reach $\$ 18$ billion in 2014 and the mobile collaborative gaming industry is seen as an area with great potential [35].

\section{The freegaming concept}

Dance Dance Revolution from Konami [3] was released in 1998 and can be regarded as the first major success of indoor exergaming as it can be played through the dance pads. The Wii Fit from Nintendo [29] is probably the most well known commercial example of an exergaming platform providing a series of games, such as golf, dance and football. Wii Fit was released in 2007 and sold more than 21 million of copies worldwide. Finally in 2010, all the major video games console manufacturers entered to the motion controlled games business with the release of Microsoft Kinect [23] and Sony PlayStation Move [38] (Fig. 1).

Indoor exergaming applications rely on virtual environments as the main game environment. The players in these types of exergames are represented in the game environment by a virtual representative (avatar). In such settings, the exergaming designer has several ways to integrate a player's exercise with the game. In a living room it is relatively easy to capture the player's motion, for example through cameras [22] and other sensors, and use this data to manipulate the avatar to mirror the player's movements. Also, the feedback can be delivered through haptic interfaces and screen facilities, while, being based on virtual artefacts, game's scenarios and objects/virtual characters surrounding the user's avatar can be easily modified and driven.

The aim of Freegaming ${ }^{1}[14]$ is to extend this exergaming concept to the outdoors. It aims to use its player's own body as an interaction interface for the game instead of a virtual avatar. This can allow integrating the exercise in a more natural way to the player's other daily activities which can consequently improve the associated health benefits. The Freegaming concept enables generating play anywhere and anytime games within a collaborative exergaming environment. The mobile augmented reality [4] aspect in Freegaming works with the smart phones' built-in GPS sensors and cameras. It uses a feature detection algorithm and more details about this approach is given in Section 2.4.

\footnotetext{
${ }^{1}$ Further information on the Freegaming project is available at http://freegaming.ucd.ie.
} 
The characteristics of Freegaming are:

\begin{abstract}
Mobile - Freegaming allows outdoor and ubiquitous exergaming. While indoor exergaming creates natural limits on what exercises can be performed, adding mobility to the exergames can open up the opportunity for encouraging many type of different exercises.

Augmented - Freegaming gives the ability to game developers to utilize augmented reality (AR) thus enabling a distinct break away from the traditional avatar paradigm. This allows the player to feel an immersive experience where it is their own physical body which interacts with the game instead of a mediated experience through a virtual avatar [20].

Collaborative - Freegaming architecture is designed to allow multiplayer gaming. It offers a suitable system to combine the socialisation feature of mobile collaboration games with the positive effects of exergames on physical health.

Adaptive - Freegaming seeks to enable outdoor exergaming. The objective of the Freegaming architecture is to adapt to this constantly changing outdoor environment.
\end{abstract}

\title{
2.1. Related research
}

Two major related areas for outdoor exergaming are AR outdoor gaming and location-aware mobile gaming. The following gaming systems were identified as exergaming systems primarily because locomotion is an essential factor to their gaming design.

AR Quake [34] is one such a game that uses GPS and a digital compass with a mobile phone, enabling players engage in battle through SMS messages. Real Tournament [50] is another application that equips every player with a PDA which has an electronic compass and GPS. It enables shooting between players through a wireless card enclosed in a water pistol shell. Human Pacman [8] is an applications where players wear HMD in outdoor gaming. However, players must carry the notebook, with its heavy batteries, in their backpacks.

There are various types of applications addressing location-aware mobile gaming. Pirates! [39] is an early application whose players use a PDA to play through WiFi networks in a few connected rooms. Players need to travel (sail) to the rooms (islands) and search for virtual resources. CatchBob! [30] is a collaborative mobile game designed for a campus area, where players carry a TabletPC. They share a map of the game zone and are capable of synchronized tagging/writing on the map; GPS is used to determine the distance of players and virtual treasure.

"Can you see me now?" [6] is a collaborative mobile game which provides a mixed real and virtual gaming experience. The online players can see their characters on a map and they can control the character with arrow keys to avoid being caught by fellow players. Their fellow players are actors that are carrying GPS and PDAs that show the game map and status; however, this game is played in a real city.

As AR outdoor and location-aware games, none of these games promise to fulfil the potential of exergaming and to achieve any health benefits. They do not contain any kind of health monitoring features, and their main objective is supplying entertainment, rather than motivating exercise, for their players.

\subsection{Challenges}

The exercise part of any exergames must satisfy certain requirements for realizing its full potential, for example, supplying personalized warm up and cool down phases [7,26,37]. As an exergame, Freegaming should be able to supply games that can be personalized to the requirements of its players. 
For outdoor exergaming, matters are definitely more complicated as it is a complex and unpredictable environment. An outdoor exergame will be played in a real world instead of in a virtual world that can be easily controlled by the game as per the deign of the game. An outdoor exergaming system must operate robustly in complex, dynamic environments. The game designer must know a lot of contextual information concerning the environment and the status of the player in this environment. In such a situation, the player's context can only be captured via sensing the player's real surroundings. Sensor data should supply two main types of information for the game concerning:

1. The players' body which includes physiological data;

2. The environment and players' status within it.

\subsection{Agent-based adaptivity}

Using sensors to collect information is not enough by itself. In addition to this, an outdoor exergame should be able to adapt itself according to the information coming from sensors. To realise this, it is necessary that the architecture can achieve adaptive and intelligent behaviour [2,17]. Intelligent agents can be used easily to integrate multiple sensors for this aim as they can form context models, and reason about them to take decisions that will affect the game. Agents have been successfully deployed in many mobile computing domains including tourism [33], mobile commerce $[19,46]$ and in outdoor exergaming environments [15].

\subsubsection{Agent Factory Mobile Edition (AFME)}

AFME [27] was used during the development of the Freegaming platform as a tool to develop agentbased mobile games. Agent Factory [9] is an open source framework that has been developed within University College Dublin (UCD) to support the development, visualization and deployment of multiagent systems. It is broadly compliant with the Foundation for Intelligent Physical Agents (FIPA) [12] standards. It is an evolving project and over the years has gone through a metamorphosis process, producing sibling frameworks such as Agent Factory Micro Edition (AFME).

AFME is a minimized footprint version of the Agent Factory platform. It is developed specifically to enable the creation of intentional agents for resource constrained devices. AFME is implemented in Java 2 Platform, Micro Edition (J2ME). In AFME, functionalities are based on the notion of several perceptors and actuators. Perceptors generate beliefs about the current state and the environment. According to this information and its internal rule set, agents decide on the plans or actions that need to be performed at a given time.

\subsection{Outdoor feature detection}

Research on robust outdoor feature and scene detection is very active [18] as it is a fundamental problem for outdoor augmented reality applications. In order to detect, identify and recognize objects in outdoor scenes, a scheme is required to find matches between features in the image that is newly taken and the images in the database. Local feature matching has become a commonly used method to compare images. Recent approaches include, but are not limited to, Scale-Invariant Feature Transform (SIFT) [21], GLOH [24], and Speeded Up Robust Features (SURF) [5].

The algorithm that is used in Freegaming is SURF which has been demonstrated to be the fastest and most accurate method to find correspondences between images [5]. Other research also shows that two variants of SURF, called U-SURF and SURF-128, outperform the other algorithms in terms of accuracy and speed [43]. 


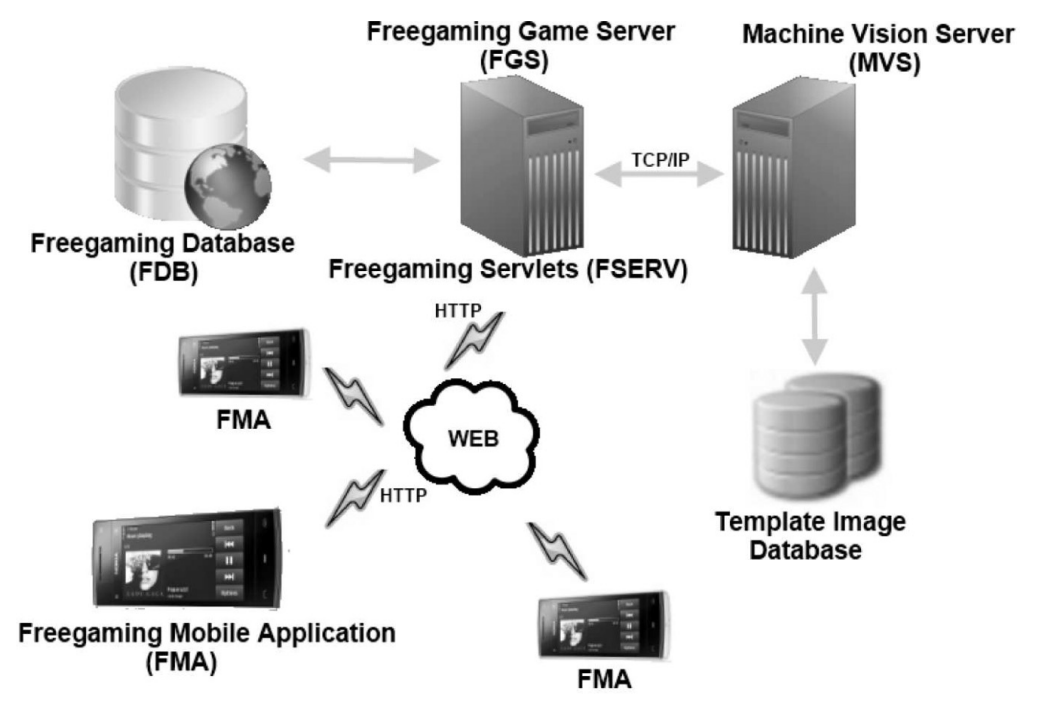

Fig. 2. A high level architectural overview of the FreeGaming platform - Game Server (FGS) incorporates the game database (FDB) and hosts the servlets (FSERV) which enable communication between server and mobile application (FMA) over the Internet. Machine Vision Server (MVS) is responsible for SURF calculations and stores the template images for this purpose. FMA running on the mobile phone communicates to the FGS by using a wireless internet connection.

\subsubsection{Speeded Up Robust Features (SURF) algorithm}

SURF algorithm is a blob detector that uses the determinant of the Hessian matrix of an approximation of the Laplacian of Gaussian to ascertain the interest points. These interest points are then described using a sixty four dimension feature descriptor devised from Haar-Wavelet responses in the position and scale at which the feature was detected. In this work the orientation of the descriptor was not important, as the camera was fixed, and therefore upright SURF (U-SURF) was used. The resultant descriptors are invariant to scale, brightness and contrast; they are not invariant to affine transformations. The software library used for this work was developed by Chris Evans [11].

Once the descriptors for the scene have been calculated they have to be compared to the descriptors in the model images so that a potential image match may be arrived at. The technique employed in this work is to use nearest neighbour matching [25]. In this approach the Euclidean distance between the best match and second best is calculated and if their ratio is less that the threshold then the best match descriptor is added to the list of descriptor matches. If the number of descriptor matches is above a certain value, then it is determined that the model is present in the scene.

As SURF is not affine invariant it is necessary to store in the database several images of the objects from different viewpoints. In Freegaming we do not segment the objects from their background.

\section{Platform design and architecture}

A Freegaming platform includes two main components. The first one is Freegaming Server and the other one is Freegaming Mobile Application (FMA) which runs on the mobile devices as a client application. Freegaming Server consists of two separate servers which are Freegaming Game Server (FGS) and the Machine Vision Server (MVS). A high level system architecture diagram for the Freegaming Platform may be seen in Fig. 2. 


\subsection{Freegaming Game Server (FGS)}

FGS includes the Freegaming Database (FDB) which maintains the game status and holds all the necessary information for each players during the game, that is, points collected, distance travelled, location, and so on. FGS also incorporates the Freegaming Servlets (FSERV) which allows the FMA to access and modify the game databases.

\subsection{Freegaming Machine Vision Server (MVS)}

Freegaming seeks to promote fitness requires so it creates incentives for a player or players to complete their goals within the game. To achieve this, the Freegaming architecture allows the developers to augment on planar surfaces in the photos taken by the mobile phone camera. This creates an Augmented Reality environment which allows the further integration of a player's physical body instead of an avatar, unlike other approaches to exergaming. The players are able to use their mobile phone as a window into a world where the virtual objects are coexisting with their physical counterparts. To generate the augmented photos, the SURF algorithm is utilised; features can be tracked from a template image onto an image taken by the phone. As the phone processor is not computationally sufficient to achieve this goal within a usable time frame, the MVS on the Freegaming Server is utilised.

\subsection{Freegaming mobile application}

FMA also has two main parts - the J2ME application and the Game Agent (GA). The J2ME application is mainly responsible for the GUI and augmentation of captured images on the mobile device screen. It includes the necessary screens for the game, that is, map view, objectives menu and augmented image screen.

The GA makes decisions concerning the game status and scenario which directly effects the J2ME application. In the current version, the GA continuously reads the Global Positioning System (GPS) data during runtime and updates its belief set concerning the state of the player and the game itself. It uses this belief set to make logical commitments for the game play, for example, identifying those zones into which the player should move. In this case, the J2ME application would generate an audio alert and show the new objective to the player.

\subsection{Implementation}

During q game, locations of all players are obtained from built-in GPS receivers on the mobile devices. GA in FMA uses these data to continuously update the game scenario. FMA communicates with FGS via FSERV and accesses the FDB or makes updates on it if necessary. Also, the images captured by mobile phone during the game are sent to the FGS and it delivers these images to the MVS for necessary SURF calculations on the images. The response coming from MVS is then reflected to the FMA via FSERV. J2ME application in FMA uses these data to augment the virtual objects on the planar surfaces over the photo taken by the player.

All communications between the Freegaming components occur over HTTP and TCP/IP connections. Communication between the FMA and the FGS occurs over either a wireless internet connection or a 3G connection where it is possible. A dataflow diagram illustrating the key communication paths between components of the Freegaming Platform may be seen in Fig. 3. 


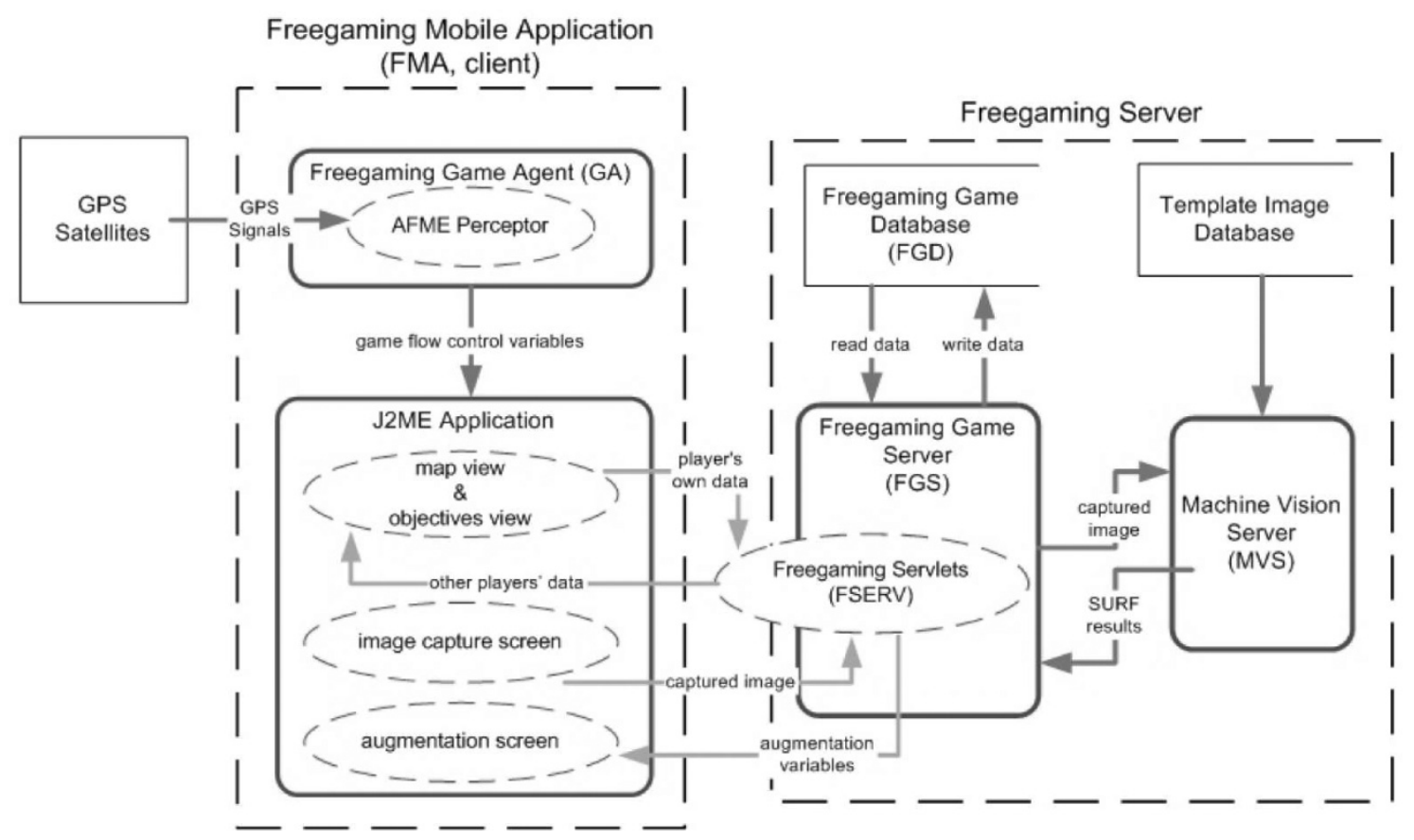

Fig. 3. A dataflow diagram of the Freegaming Platform that illustrates how data is interchanged between the key Freegaming Platform components.

The Freegaming platform adopts the intelligent agent paradigm and is implemented in practice by both Agent Factory and Agent Factory Mobile Edition (AFME). Agent Factory is being harnessed for the fixed networked components. It is envisaged that the availability of sophisticated reasoning engines on both elements will maximise the opportunities for delivering adaptive, personalised games. Specifically, the FMA has been coded using AFME's BDI-style agent which allow FMA to use any sensor data to adopt itself both to players' body information and also information concerning the environmental context.

\section{Game creation}

The Freegaming platform is designed to allow game designers to generate location sensitive exergames. It allows designers to create location-based objectives for the game. Because of this, game developers need to define special regions in a pre-defined gaming area. These special regions form the objective list for GA and the players in the game. All special regions will have their related GPS coordinates defined in the GA which will control the game flow during runtime. In the current version the GPS sensor data is used for different adaptations during the game play. The simplest one is the adaption of the graphical user interface on the phone screen. For example, players need to arrive in specific GPS locations to take snapshots of the scene. The game will not show the button for taking snapshots until the players physically reaches the target GPS location. After reaching the target location, GA will update the necessary information for the FMA; afterwards, players will see their new objective on their phone screen, and the map view will be updated according to these changes in the game scenario.

At the same time, game designers need to generate a template images database for each special region. These template images will be used in the MVS for SURF calculations to decide where to make 


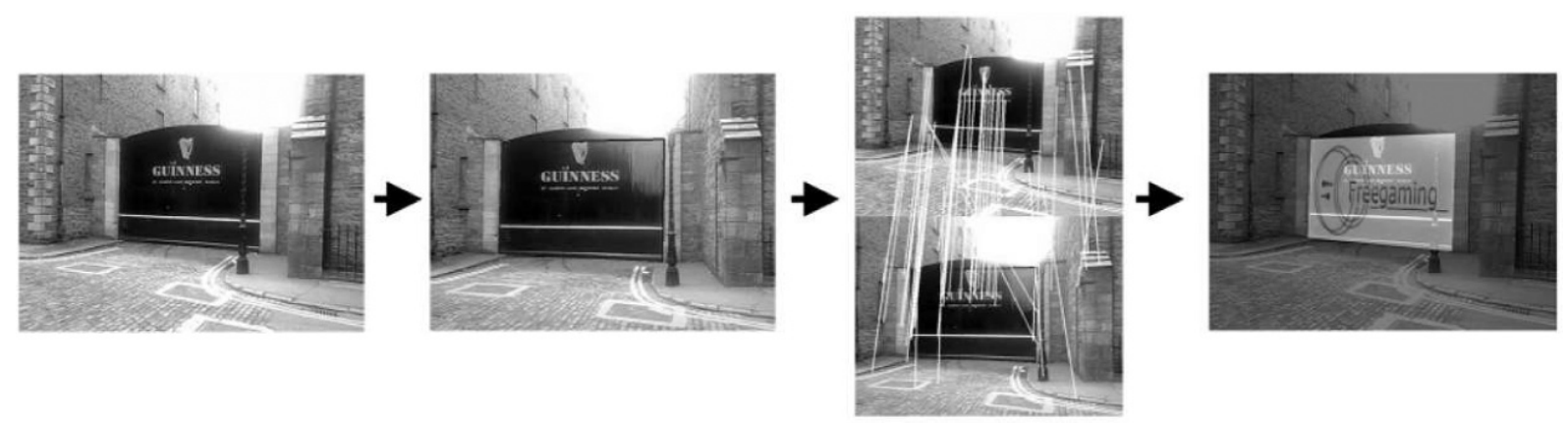

Fig. 4. Augmented Reality screen shots from Freegaming Machine Vision Server showing, from left to right 1) the original image; 2) the result of feature detection; 3) SURF calculation points are highlighted; 4) the augmented photo generated by MVS by using the original image and the SURF calculations.

the suitable augmentation on the captured image (Fig. 4). To generate these image databases, game developers need to take photographs of pre-defined planar surfaces in the special regions. Then, these surfaces should be tagged in the photographs by the game developers. The Freegaming platform has a separate simple tagging program (coded by Qt programming language) which allows the programmer to tag planar surfaces taken from photographs within the game area. During the game, when a player reaches a target location the next objective is generally taking a photo of a certain object inside this location. After capturing, player will wait for a response from the server. During this time the captured photo is sent to MVS and it uses the template image databases to analyse the newly captured image and decide on where to augment the virtual objects.

Freegaming platform shows the potential of Agent-based and AR-based design to increase the flexibility while generating a game. Agent-based design eases the implementation of different games based on the same platform. In the Freegaming platform, agents are used to integrate and manage different resources, for example different sensors, and interact with services provided by the extended infrastructure, for example localization services. Changing the initial belief set, the goals of the agent and/or its commitment rules and also changing the virtual AR content will result with a totally different game.

\subsection{Game implementation}

To implement the Freegaming Platform's capabilities, a prototype game is generated. This is a proof of concept game and the full promise of the platform can only be obtained by integrating specific content. This prototype is not using the multiplayer functionality and can only be played as a single player game. The screenshots in Figs 5 and 6 display this game as it is implemented on a Nokia N97. There are two special regions in this game and four objectives.

At the beginning of the game, player enters his/her age. After this, the player sees a start menu for the game where they can either select to see instructions about how to play the game or start a new round for the game. When the game begins, players can see the map view on their mobile phone screen; this shows the player icon overlaid on the map of a gaming area. For the first objective, player needs to go into the first region which is the starting point for the game. When the player reaches this point, GA detects this automatically by reading the GPS signal and automatically changes the player's state in the game. At this point, the player is prompted with a sound and a visual alert on their mobile screen about a new objective. This second objective is to take a picture of the scene, especially the walls or windows. When the player captures a proper picture of the scene via their mobile phone camera, MVS detects 

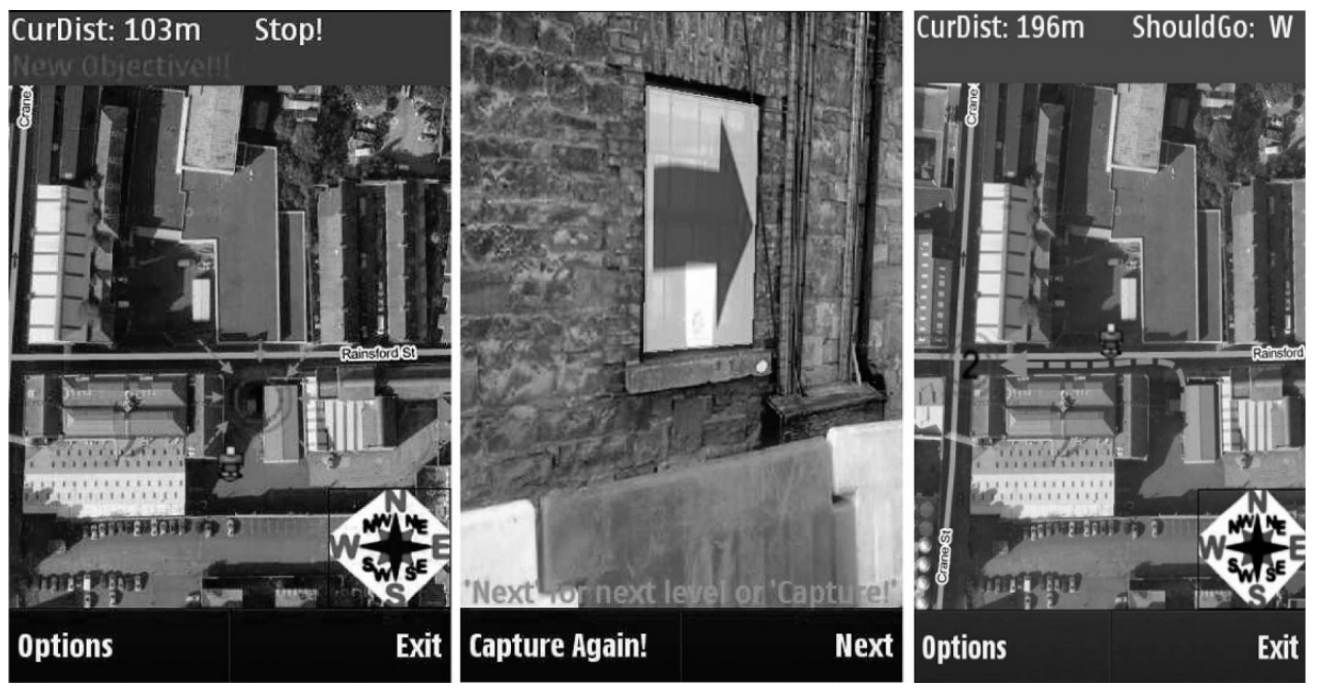

Fig. 5. Sample screen shots from Freegaming Mobile Application's Map View and Augmentation screens. 1) The players see the target location and their icon relative to their GPS location in the Map View, it can be seen that the player is very close to the target point. 2) When the player reaches a target location, the game asks for a photo capture. The game superimposes an arrow onto the captured image which shows the direction for the next target location. 3) the J2ME application and the Map View screen are updated by the Game Agent and it now shows the new target on the screen.
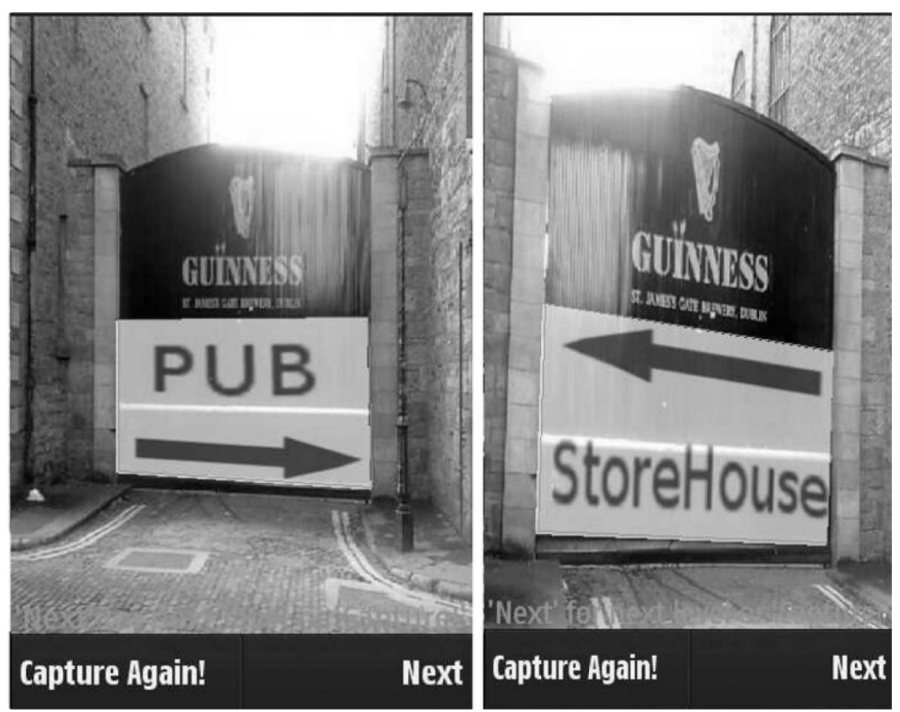

Fig. 6. Agent controlled augmentation - the Game Agent can augment different visual objects on the images based on personalization data. 1) the player is older than 21 and the game augmented a direction for the nearest pub. 2) the player cannot buy alcohol legally; thus the game augments an arrow which shows the direction for a shop.

the windows inside this photo and augments virtual arrows on them that shows the direction of the next target location. After achieving the second objective, the map view of the game is also updated by the GA and the player can see where to go for the next target location on the map (Fig. 5). When the player reaches the second target zone, the game again raises an alert about the new image capturing objective. This time, the game asks for a picture of the gate in the target area. When the player takes the picture, 
GA decides on which message to augment on the gate based on the personalization information given by the player at the beginning of the game. It augments the direction for the nearest pub if the player is over 18 or the direction for the storehouse if the player is under 18 years old (Fig. 6). After that, the game informs the player that this round of the game is finished.

\section{Future developments}

Mobile Augmented Reality [10] offers an exciting basis for a new generation of Exergaming platforms. As an illustration of the possibilities, a futuristic game scenario is now presented after which the technologies necessary to realise this vision are considered.

\subsection{A futuristic game scenario}

The number of mobile phone subscribers is increasing rapidly. Think about a space where sensors are placed everywhere and different technologies work together to help realize the vision of ubiquitous computing [47]. This concept proposes that sensors be placed everywhere, even in the body, and that seamless interaction with this embedded infrastructure is possible. It is envisaged that a combination of sensor networks and mobile phones will converge, giving rise to a new generation of services, including games.

John's Calorie Bill

John was feeling a little hungry and ate a chocolate bar before leaving home for college. As he is currently on a diet, he informs the application running on his mobile phone about his additional food intake. Agents in this mobile application are in charge of his diet and suggest a mobile game to help him burn those additional calories. It outlines a game in which John has to find a hidden treasure using a virtual treasure map of the real world around him. The agent has set the final destination as John's college. John is also wearing one of his new shirts which contains a heart monitor. As the game starts, John is shown an overhead map pointing him to the end of the road. John proceeds briskly, looking occasionally at his mobile phone. He reaches the first destination point and is told that in order to get to the next location, he has to take a left at the palm tree. John knows that there is no palm tree in the physical world, so he picks up his phone and takes a picture of the junction ahead of him. The photo taken on his camera is augmented with an image of the virtual world. Through this magic mirror he sees a palm tree placed at the corner of the road. Knowing his new direction he quickly heads down the road. As John's agent senses that his heartbeat has not increased sufficiently for a good workout, it sets the next target on top of a hill just outside the college. After this location, John finally arrives in college. The final destination is placed just outside his Department, and John takes a picture of the front door believing this to be the location of the virtual bounty. The image returned contains only simple text, however, telling him the treasure is hidden in the place that others would just waste. Looking around, he notices a waste bin and takes another picture. The image appears with a virtual treasure chest replacing the waste bin, and the mobile application congratulates him.

\subsection{Ongoing research}

At present, the Freegaming prototype described above is far from the application mentioned in previous section but it still implements a basic exergaming platform which can be improved. The current 
architecture of Freegaming allows us to give full control of the game to the GA running on the phone. As it already includes a GPS sensor, future integration of Heart Rate (HR) and accelerometer sensors are significantly less challenging to implement given the flexibility of the agent abstraction. Also, agents can use personal information like age, height, and so on. The game must be seeded with this information prior to commencement if a fully personalized exercise regime is to be dynamically constructed, and the user interface is to adapt appropriately. A simple example could be increasing the target HR for a player by defining an uphill GPS position as a target location. Agents can address any disconnections that occur during a game. In the case of weak GPS signals, the GPS monitoring agent will know when the GPS signal is lost. It can use the last estimate and increase the range of the space over time which the game considers while looking for a match to the image captured by the user. Agents can increase the range for the players which are in a location with weak GPS signals and decrease the range for the players with strong signals. Furthermore, an agent can be implemented that will periodically ping the Machine Vision server. If this server is unavailable, the GA can easily change the objectives for the game in real-time in order to hide this from the user. For example, instead of asking the user to capture a picture, which GA knows that cannot be submitted, the game can ask to cancel the current objective and reach another location, which will also increase the probability that the connection will work again. A further improvement can be creating an agent to deal with the changes in the weather and environment by adding proper sensor capabilities or virtual perceptors connected to online weather reports.

A key ongoing research goal within the Freegaming project is to create a more engaging scenario for the players. There are already documented examples on intelligent multimedia delivery and presentation using agent-based approaches and mobile devices, for example [32]. The intention in Freegaming is to expand the initial augmented reality interface from augmenting still camera frames into augmenting live video taken by the camera [13]. To achieve this goal, it is intended to utilise the accelerometer within the phone to compliment the external machine vision server. Using an external server to process the camera data requires the use of existing cellular networks which use $3 \mathrm{G}$. $3 \mathrm{G}$ protocol poses a significant challenge to the programmer as it has unpredictable data rates. An Agent-based approach could help alleviate some of these problems by using intelligent pre-caching [31]. Each phone within Freegaming can have a dedicated agent assigned the task of deducing the current orientation in relation to the previous snapshot taken. This would of course only give an approximation for the placement of the augmented realty object but would alleviate the current disruption of waiting for the image server to process the latest camera snapshot.

\section{Conclusion}

In this paper, Freegaming, an agent-based mobile collaborative exergaming platform was introduced. The design of the Freegaming platform upon which multiple Freegaming applications can be deployed is described with the implementation of a proof of concept game. Exergaming has the potential to help fight against obesity in the future. Although its primary audience would be young people who are part of a generation brought up in an electronic age, the exergaming idea can also be applicable for adults who have a sedentary lifestyle. The Freegaming platform architecture was presented and the use of agents proposed as a means of managing the inherent difficulties inherent in outdoor environments as well as enabling collaboration and coordination in arbitrary games. The concept of utilising augmented reality within exergaming allows the player a more immersive experience within the virtual world and an honest feeling of achievement for their actions instead of living vicariously through an avatar. Mobile exergaming provides an opportunity to make computer games more fun, more engaging and, crucially, 
live up to their potential as a medium that can enrich both mind and body. In this way players enjoy the rich medium of computer games for health benefits rather than encouraging lifestyles that may well compromise their future health.

\section{Acknowledgments}

This work is supported by the National Digital Research Centre(NDRC) Research Grant entitled FreeGaming Project number SP/010 and in part by Science Foundation Ireland under grant 07/CE/I1147.

\section{References}

[1] ACT for Youth Center of Excellence: Research facts and findings: Childhood obesity, http://www.actforyouth.net/ resources/rf/rf_obesity_0603.pdf (2003). Accessed 12 January 2012.

[2] J. Ahn, Novel directory service and message delivery mechanism enabling scalable mobile agent communication, Mobile Information Systems 4(4) (2008), 333-349.

[3] G. Andrews, Dance dance revolution, in: Space Time Play, F. Borries, S.P. Walz and M. Böttger, eds, Birkhäuser Basel, 2007, pp. 20-21.

[4] R. Azuma, M. Billinghurst and G. Klinker, Editorial: Special section on mobile augmented reality, Computers and Graphics 35 (2011), vii-viii.

[5] H. Bay, T. Tuytelaars and L.J. Van Gool, SURF: Speeded up robust features, in: Computer Vision - ECCV 2006, Lecture Notes in Computer Science, vol. 3951, Springer 2006, pp. 404-417.

[6] S. Benford, A. Crabtree, M. Flintham, A. Drozd, R. Anastasi, M. Paxton, N. Tandavanitj, M. Adams and J. Row-Farr, Can you see me now? ACM Trans Comput Hum Interact 13 (2006), 100-133.

[7] I. Bogost, The rhetoric of exergaming, in: Proceedings of the Digital Arts and Cultures (DAC), USA, 2005.

[8] A. Cheok, K. Goh, W. Liu, F. Farbiz, S. Fong, S. Teo, Y. Li and X. Yang, Human pacman: a mobile, wide-area entertainment system based on physical, social, and ubiquitous computing, Personal and Ubiquitous Computing 8 (2004), 71-81.

[9] R. Collier, G.M.P. O'Hare, T. Lowen and C. Rooney, Beyond prototyping in the factory of agents, in: Proceedings of the 3rd Central and Eastern European conference on Multi-agent systems, CEEMAS'03, Springer-Verlag, Berlin, Heidelberg, 2003, pp. 383-393.

[10] C. Endres, A. Butz and A. MacWilliams, A survey of software infrastructures and frameworks for ubiquitous computing, Mobile Information Systems 1 (2005), 41-80.

[11] C. Evans, Notes on the opensurf library. Tech. Rep. CSTR-09-001, University of Bristol (2009), http://www.cs.bris. ac.uk/Publications/Papers/2000970.pdf. Accessed 12 January 2012.

[12] FIPA: The foundation for intelligent physical agents - standard status specifications, http://www.fipa.org/repository/ standardspecs.html (2000). Accessed 12 January 2012.

[13] T. Gao, G. Li, S. Lian and J. Zhang, Tracking video objects with feature points based particle filtering, Multimedia Tools and Applications (2011), 1-21.

[14] L. Görgü, A.G. Campbell, K. McCusker, M. Dragone, M.J. O’Grady, N.E. O’Connor and G.M.P. O'Hare, Freegaming: mobile, collaborative, adaptive and augmented exergaming, in: Proceedings of the 8th International Conference on Advances in Mobile Computing and Multimedia, MoMM '10, ACM, New York, NY, USA, 2010, pp. 173-179.

[15] L. Gorgu, G.M.P. O'Hare and M.J. O'Grady, Towards mobile collaborative exergaming, in: Advances in Human-oriented and Personalized Mechanisms, Technologies, and Services, 2009. CENTRIC '09. Second International Conference on, 2009, pp. 61-64.

[16] J. Hoysniemi, International survey on the dance dance revolution game, ACM Computers in Entertainment 4(2) (2006), 3-30.

[17] I.K. Ibrahim, Norms for modeling agents' interaction in ubiquitous environments, Mobile Information Systems 4(1) (2008), 69-76.

[18] Y. Kalantidis, G. Tolias, Y. Avrithis, M. Phinikettos, E. Spyrou, P. Mylonas and S. Kollias, Viral: Visual image retrieval and localization, Multimedia Tools and Applications 51 (2011), 555-592.

[19] S. Keegan, G.M.P. O'Hare and M.J. O'Grady, Easishop:ambient intelligence assists everyday shopping, Information Sciences 178(3) (2008), 588-611.

[20] T. Kosutic, M. Mosmondor, I. Andrisek, M. Weber, M. Matijasevic and I.S. Pandzic, Personalized avatars for mobile entertainment, Mobile Information Systems (2006), 95-110. 
[21] D. Lowe, Distinctive image features from scale-invariant keypoints, International Journal of Computer Vision 60 (2004), 91-110.

[22] R. Marks, Eyetoy, innovation and beyond (2010). http://blog.us.playstation.com/2010/11/03/eyetoy-innovation-andbeyond/comment-page-2/\#comment-478157. Accessed 12 January 2012.

[23] Microsoft: Introducing kinect for xbox 360. http://www.xbox.com/en-US/kinect (2011), Accessed 12 January 2012.

[24] K. Mikolajczyk and C. Schmid, Performance evaluation of local descriptors, IEEE Trans Pattern Anal Mach Intell 27 (2005), 1615-1630.

[25] K. Mikolajczyk and C. Schmid, A performance evaluation of local descriptors, Pattern Analysis and Machine Intelligence, IEEE Transactions on 27(10) (2005), 1615-1630.

[26] F.F. Mueller and S. Agamanolis, Exertion interfaces, in: CHI '08 extended abstracts on Human factors in computing systems, CHI EA '08, ACM, New York, NY, USA, 2008, pp. 3957-3960.

[27] C. Muldoon, G.M.P. O'Hare, R.W. Collier and M.J. O'Grady, Towards pervasive intelligence: Reflections on the evolution of the agent factory framework, in: Multi-Agent Programming, A. El Fallah Seghrouchni, J. Dix, M. Dastani and R.H. Bordini, eds, Springer US, 2009, pp. 187-212.

[28] National Heart Lung and Blood Institude (NHLBI) Obesity Education Initiative: The practical guide to the identification, evaluation and treatment of overweight and obesity in adults. http://www.nhlbi.nih.gov/guidelines/obesity/prctgd_c.pdf (2000). Accessed 12 January 2012.

[29] Nintendo: What is wii fit plus. http://wiifit.com/ (2011). Accessed 12 January 2012.

[30] N. Nova, F. Girardin and P. Dillenbourg, 'location is not enough!': an empirical study of location-awareness in mobile collaboration, in: IEEE International Workshop on Wireless and Mobile Technologies in Education (WMTE 2005), 2005, pp. 21-28.

[31] M.J. O'Grady, G.M.P. O’Hare and R.W. Collier, Mobile multimedia: Reflecting on dynamic service provision, International Journal of Ambient Computing and Intelligence 2(3) (2010), 19-39.

[32] M.J. O'Grady, G.M.P. O'Hare and C. Donaghey, Delivering adaptivity through context-awareness, Journal of Network and Computer Applications 30(3) (2007), 1007-1033.

[33] M.J. O'Grady, G.M.P. O'Hare and C. Sas, Mobile agents for mobile tourists: A user evaluation of gulliver's genie, Interacting with Computers 17(4) (2005), 343-366.

[34] W. Piekarski and B. Thomas, Arquake: the outdoor augmented reality gaming system, Commun ACM 45 (2002), 36-38.

[35] L. Portela, J. ten Sythoff and S. Boguszewska, Mobile gaming in emerging markets five-year forecast and impact analysis, http://www.pyramidresearch.com/store/RPMOBILEGAMING0907.htm (2009). Accessed 12 January 2012.

[36] V.W. Setzer and G. Duchett, The risks to children using electronic games. http://www.ime.usp.br/ vwsetzer/video-grisks.html (2006). Accessed 12 January 2012.

[37] J. Sinclair, P. Hingston and M. Masek, Considerations for the design of exergames, in: GRAPHITE '07: Proceedings of the 5th international conference on Computer graphics and interactive techniques in Australia and Southeast Asia. ACM, Australia (2007).

[38] Sony Computer Entertainment America LLC: Move into the action. http://us.playstation.com/ps3/playstation-move/ (2011). Accessed 12 January 2012.

[39] B. Staffan, F. Jennica, H. Rebecca and L. Peter, Pirates! using the physical world as a game board, in: Human-Computer Interaction INTERACT01. Japan (2001).

[40] W. Tanson, Adolescent obesity largely caused by lack of physical activity. http://www.unc.edu/news/archives/apr03/ sutherland040903.html (2003). Accessed 12 January 2012.

[41] The Obesity Society: Obesity statistics. http://www.obesity.org/resources-for/obesity-statistics.htm (2007). Accessed 12 January 2012.

[42] U.S. Department of Health and Human Services: The surgeon general's call to action to prevent and decrease overweight and obesity. http://www.surgeongeneral.gov/topics/obesity/calltoaction/CalltoAction.pdf (2001). Accessed 12 January 2012.

[43] C. Valgren and A. Lilienthal, Sift, surf and seasons: Long-term outdoor localization using local features. in: Proc. European Conference on Mobile Robots (ECMR), 2007, pp. 253-258.

[44] E.A. Vandewater, M. Shim and A.G. Caplovitz, Linking obesity and activity level with children's television and video game use, $J$ of Adolescence 27(1) (2004), 71-85.

[45] V. Vasudevan, Collaborative mobile gaming (2007), http://www.motorola.com/staticfiles/Consumers/Corporate/USEN/_Documents/technology_vasudevan_collaborative-mobile-gaming.pdf. Accessed 12 January 2012.

[46] Y. Wang, D.S. Wong and H. Wang, Employ a mobile agent for making a payment, Mobile Information Systems 4 (2008), $51-68$.

[47] M. Weiser, Ubiquitous computing, Computer 26 (1993).

[48] World Health Organisation: Obesity and overweight - fact sheet 311. http://www.who.int/mediacentre/factsheets/fs $311 /$ en/index.html (2011). Accessed 12 January 2012. 
[49] World Health Organization: Diet and physical activity: A public health priority. http://www.who.int/dietphysicalactivity/ en/ (2011). Accessed 12 January 2012.

[50] M. Wu, K. Mitchell, D. McCaffery, J. Finney and A. Friday, Real tournament: mobile context-aware gaming for the next generatation, Electronic Library 22 (2004), 55-64.

Levent Görgü obtained a B.Sc. degree in Computer Engineering in 2005 from Eastern Mediterranean University (Cyprus) and a M.Sc. degree in Computer Science in 2007 from University College Dublin (Ireland). Now he is a Ph.D. student at the School of Computer Science \& Informatics at University College Dublin and studying within the CLARITY Centre for Sensor Web Technologies. He is working on his thesis "Intelligent Mobile Control over Wireless Sensor Networks", under the supervision of Prof. Gregory O'Hare. His research condenses on the application of Agent Programming to the mobile context. Specifically, his thesis focuses on the idea of using Agent-Based mobile applications to control and monitor Wireless Sensor Networks (WSN). This can be exemplified as running agents on mobile devices or sensor nodes for both WSN controlling and optimization.

Abraham G. Campbell is a PhD candidate in University College Dublin, where he previous conducted his undergraduate studies in Computer Science. His PhD thesis is on Augmented Reality Agents which is an exploration of the potential synergy of Augmented Reality and Multi-Agent Systems.

Kealan McCusker received a B.Sc. in Physics from the University of Manchester in 1994. He then obtained an M.Sc. in Electronic Engineering from Queen's University Belfast, graduating in 1996. After graduating, he worked from 1997 until 2002 for Parthus Technology Ltd and was employed as a application-specific integrated circuit (ASIC) designer where he was exposed to the full chip design cycle. In 2003 he worked as an independent contractor for Texas Instruments in Nice, France. Since 2007 he has been employed as a post doctoral researcher in the Dublin City University primarily concerned with translational research. For example, a recent project was based in the National Digital Research Centre (NDRC) and it investigated exergaming, specifically using a smart phone as the game console. His role, among other tasks, was to implement a computer vision server and object recognition on the mobile platform using the Speeded Up Robust Features (SURF) algorithm. Currently, he is investigating video and audio analysis technology to create deep, structured metadata for moving image assets.

Mauro Dragone is a Research Fellow in the CLARITY Centre for SensorWeb Technology at the School of Computer Science and Informatics, UCD. He received his PhD from UCD and a Magna Cum Laude Laurea degree in Computer Science from the University of Bologna, Italy. Prior to his involvement with the CLARITY Centre, he worked for more than 12 years in the software industry. His current research addresses a wide range of theoretical and practical aspects of software engineering and artificial intelligence for robotics, ubiquitous systems and wireless sensor networks.

Michael J. O'Grady is Post doctoral Researcher at the School of Computer Science \& Informatics at University College Dublin. His research interests include Ambient Intelligence, Mobile Computing and Multimedia Systems. Prior to commencing a PhD in 1999, he spent almost a decade working in the software and telecommunications industries. In his research career, he has authored and contributed to over 100 publications, and has served in a variety of roles in conference organisation and review committees. Dr. O'Grady is a senior member of the ACM and IEEE. He is a member of the editorial board of the Journal Of Ambient Intelligence And Humanized Computing.

Noel E. O'Connor graduated from Dublin City University (DCU) with a B.Eng. in Electronic Engineering (1992) and a PhD (1998). He is currently an Associate Professor in the School of Electronic Engineering at DCU and a Principal Investigator (PI) in CLARITY: Centre for Sensor Web Technologies. His early research was in the field of video compression, specifically object-based compression in the context of MPEG-4, which subsequently led to an interest in video object segmentation and tracking as well as other aspects of computer vision. With the advent of MPEG-7, he became interested in audio-visual (AV) analysis for content-based information retrieval as well as low-power configurable hardware for AV processing, a key-enabler for next generation context-aware multimedia sensors. He has a long track record of contributing to international standardization. The focus of his current research is in multi-modal content analysis leveraging mutually complementary sensor data sources, for applications in sports and health, entertainment, ambient assisted living and environmental monitoring. He has edited 6 journal special issues, including Signal Processing: Image Communication, Multimedia Tools and Applications, the Journal of Web Semantics and the Journal of Embedded Systems. He has acted as Programme Co-Chair for 4 international conferences, CIVR 2009, SAMT 2006, WIAMIS 2007 and CIVR 2004. He was Content Track Chair for ICME 2008 and Tutorial/Workshop Chair for SAMT 2009, CBMI 2009 and SAMT 2008. He is a member of the Academic Steering Committee of the ACM Multimedia Grand Challenge series, run in conjunction with ACM Multimedia. He is a member of the IEEE.

Gregory M.P. O'Hare is a Professor at UCD. He has published over 320 refereed publications in Journals and International Conferences. His research interests are in the areas of Distributed Artificial Intelligence and Multi-Agent Systems (MAS), and 
Mobile \& Ubiquitous Computing, Autonomic Systems, Robotics, and Wireless Sensor Networks. O'Hare is a Fellow of the British Computer Society a member of the ACM, AAAI and a Chartered Engineer. He also held a prestigious Science Foundation Ireland (SFI) Principal Investigator Award 2003-2007, and one of the Principal investigators and founders of CLARITY. Prof. O'Hare serves the editorial board of a number of international journals including the International Journal on Knowledge Based Intelligent Engineering Systems. 

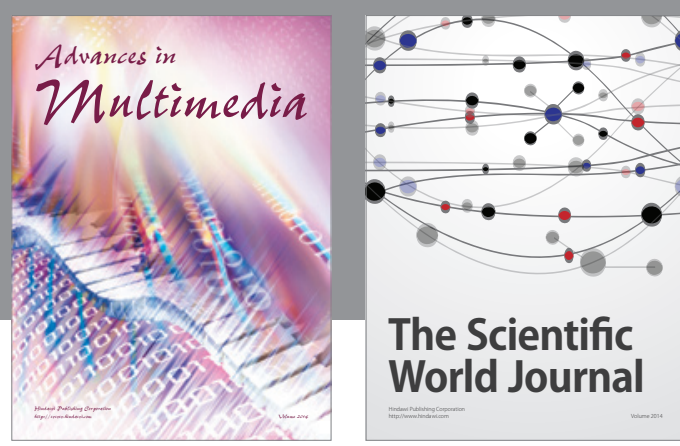

The Scientific World Journal
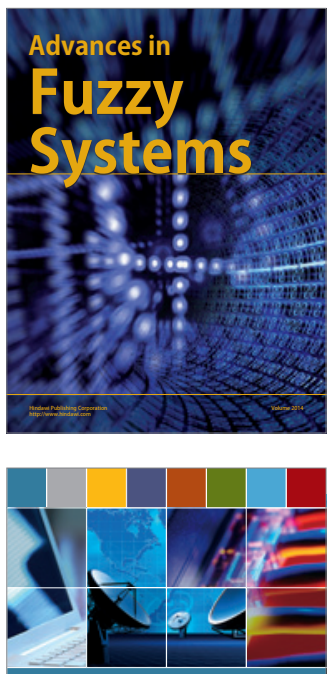

Computer Networks and Communications
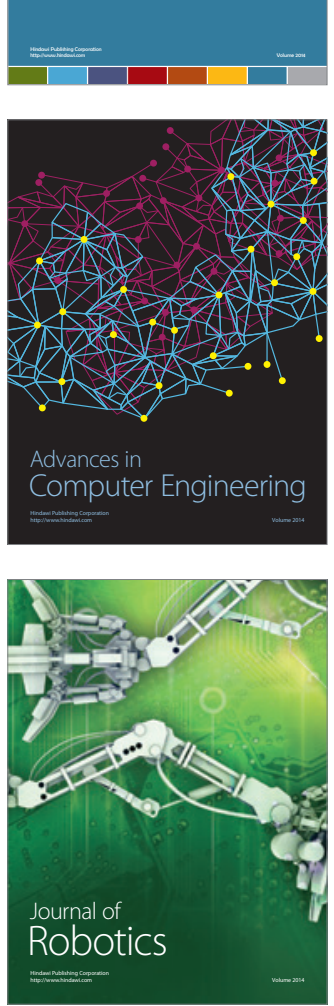
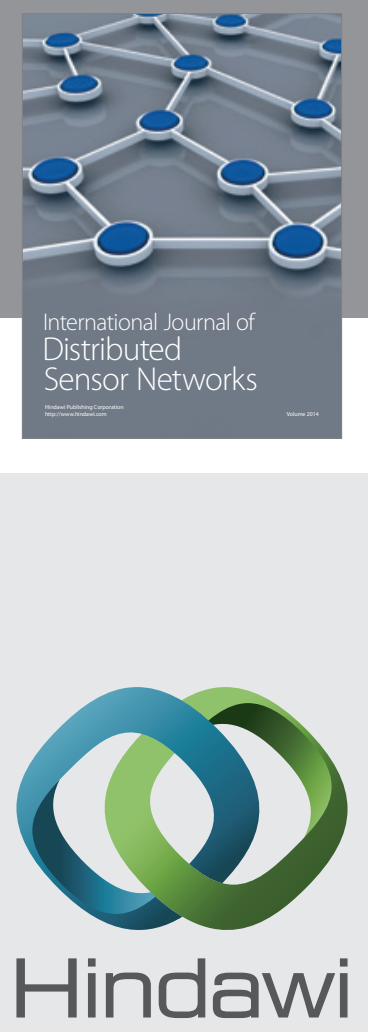

Submit your manuscripts at

http://www.hindawi.com
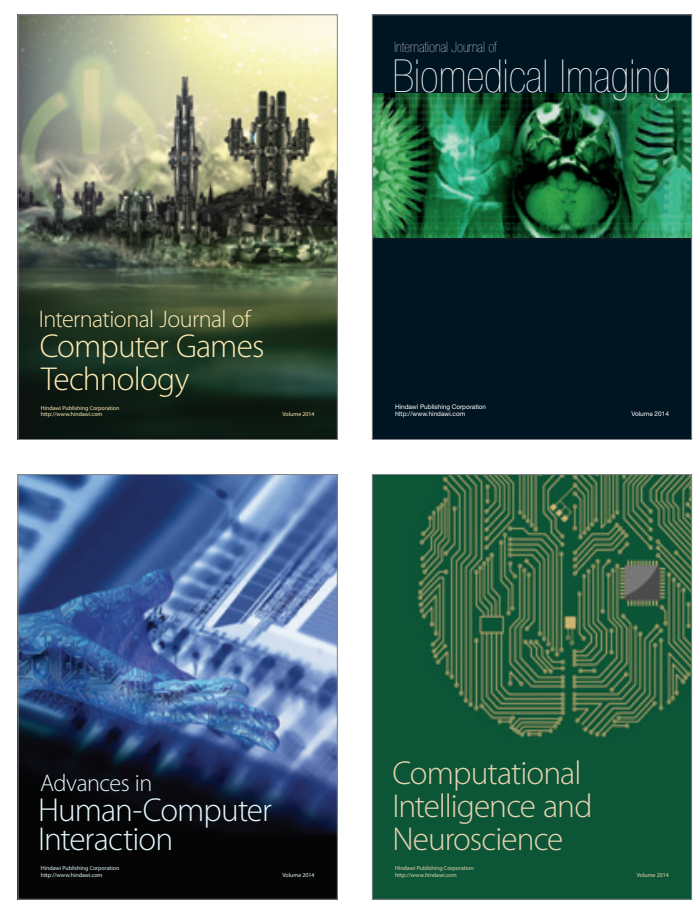
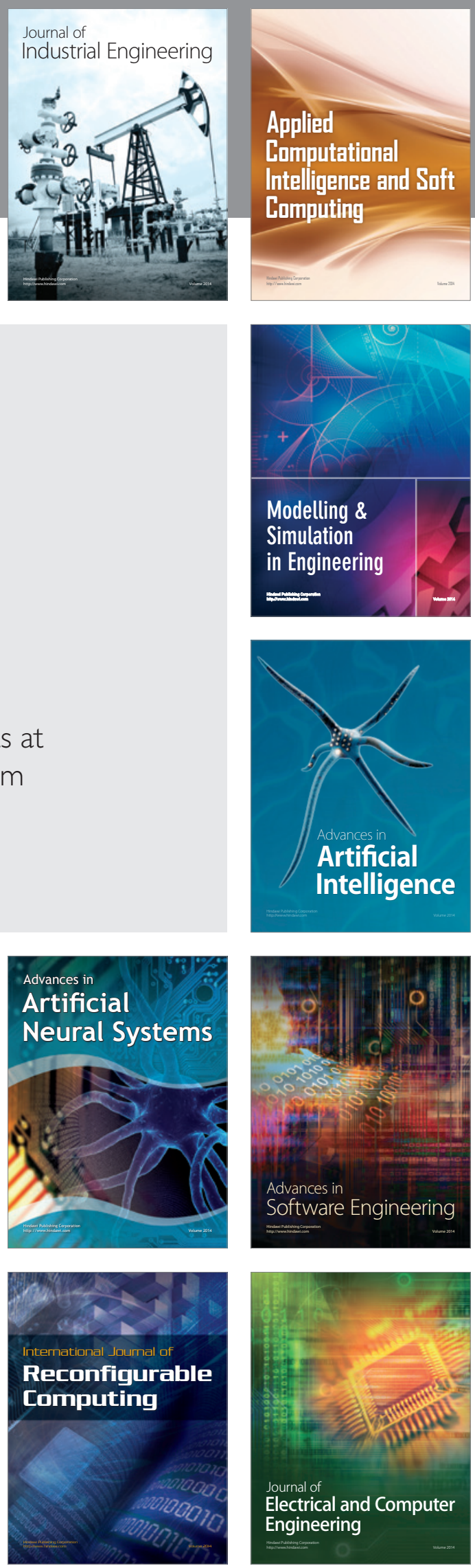